\title{
Revisión literaria sobre los factores que inciden en la competitividad de las empresas
}

Revisão literária sobre os fatores que afetam a competitividade das empresas

A review from literature about the factors influencing companies' competitiveness

\author{
Hector Hugo Maldonado Salinas \\ hector.mldo@gmail.com \\ Universidad Autónoma de Nuevo León - México \\ https://orcid.org/0000-0003-0386-4253
}

\begin{abstract}
RESUMEN
La entrada en vigor del T-MEC es una herramienta que permite a México impulsar su economía y adaptarla a las tendencias y necesidades que demanda la nueva etapa de la Globalización en el siglo XXI, sin embargo; la competitividad de la economía mexicana en contraste con la economía de cualquier otro país desarrollado presenta un desequilibrio en términos sociales, económicos y comerciales. La presente investigación estima como objetivo central identificar los principales factores que inciden en la competitividad de las empresas, estriba en el tipo cualitativo, no experimental, bajo una revisión exhaustiva de la literatura científica. Como resultado se obtuvo; un marco teórico/literario que servirá como referente para futuras investigaciones. A partir de la revisión literaria, se identificaron un total de ocho factores que han actuado como los determinantes que inciden en la competitividad de las empresas.
\end{abstract}

Palabras clave: Comercio Internacional, Competitividad, Empresas, México, Tratado de Libre Comercio.

\section{RESUMO}

A entrada em vigor do T-MEC é uma ferramenta que permite ao México dinamizar a sua economia e adaptá-la às tendências e necessidades exigidas pela nova fase da Globalização do século XXI; a competitividade da economia mexicana em comparação com a economia de qualquer outro país desenvolvido apresenta um desequilíbrio em termos sociais, econômicos e comerciais. A presente pesquisa tem como objetivo central identificar os principais fatores que afetam a competitividade das empresas, ela reside no tipo qualitativo, não experimental, mediante uma revisão exaustiva da literatura científica. Como resultado, foi obtido; um referencial teórico / literário que servirá de referência para pesquisas futuras. A partir da revisão literária, foram identificados um total de oito fatores que atuam como determinantes que afetam a competitividade das empresas.

Palavras-chave: Comércio Internacional, Competitividade, Empresas, México, Acordo de Livre Comércio.

\begin{abstract}
The entry into force of the T-MEC is a tool that allows Mexico to boost its economy and adapt it to the trends and needs demanded by the new stage of Globalization in the 21st century, however; the competitiveness of the Mexican economy in contrast to the economy of any other developed country presents an imbalance in social, economic, and commercial terms. The present research considers as a central objective to identify the main factors that influence the competitiveness of companies, it is based on qualitative, non-experimental type, under an exhaustive review of scientific literature, it was obtained as a result; a theoretical / literary framework that will serve as a reference for future research. From the literary review, a total of eight factors were identified that have acted as the determining factors that affect the competitiveness of companies.
\end{abstract}

Keywords: Business, Competitiveness, Free Trade Agreement, International Trade, Mexico.

\section{INTRODUCCIÓN}

La disolución del pacto de Varsovia en 1989 y la desintegración de la Unión de Repúblicas Soviéticas Socialistas en 1991 ocasionó una alteración en el Sistema Internacional, a partir de esa época las prioridades de la agenda internacional de los Estado-Nación cambiaron, la primacía pasó 
de ser un conflicto ideológico entre dos clases (capitalismo-comunismo), enfocándose en consolidar un comercio exterior firme y economía creciente.

La economía y el comercio mundial refiere la Organización Mundial de Comercio (OMC, 2016) están intrínsecamente relacionados, esto apunta que a mayor o menor crecimiento económico; mayor o menor tasa de crecimiento del comercio mundial, esto debido a que la economía es una actividad dinámica que se fundamenta en el cambio constante de los bienes y servicios mediados por la tecnología y la innovación, mismas que explican el dinamismo desmesurado que ha experimentado el comercio mundial en las dos últimas décadas

A partir de la era de la globalización los Estados-Nación optaron por integrar sus economías en bloques regionales para impulsar la economía y comercio, de esta manera en la región Norteamericana en 1992, se firma el Tratado de Libre Comercio entre México, Estados Unidos y Canadá (TLCAN), el cual entra en vigor el primero de enero de 1994, convirtiéndose en un relativo en el comercio exterior.

La entrada en vigor del TLCAN fue una herramienta que permitió a México impulsar su economía y acoplarse a las tendencias y necesidades que demandó aquella época, sin embargo; las diferencias tanto sociales, económicas y comerciales fueron colosales comparando la competitividad de la economía mexicana con la estadounidense y la canadiense, lo que ha provocado debates desde entonces entre sí fue lo correcto o no para México ingresar al tratado, como consecuencia se comenzaron a gestar ideas que permitieron reformar e impulsar la economía mexicana para equilibrar la asimetría existente.

Maldonado y Ortiz (2021) mencionan que en 2018 después de casi un cuarto de siglo de existencia del TLCAN, los gobiernos de los tres países que conformaron el acuerdo comercial acordaron sustituirlo por el Tratado entre México, Estados Unidos y Canadá (T-MEC), este tratado introdujo la regulación energética, comercio electrónico y medio ambiente, además de reestructurar los apartados del sector automotriz, textil, maquilador y manufacturero. El TMEC entró en vigor el primero de Julio de 2020, sujeto a una revisión periódica cada seis años, con el fin de garantizar la medición de resultados y el beneficio de cada una de sus actores.

Bajo este contexto, en el siglo XXI el impulso de la agenda internacional reitera que la responsabilidad de la competitividad no solo engloba la competencia de las empresas, sino que cada vez en mayor medida los actores públicos comienzan a encontrar balance y amplia responsabilidad en garantizar la competitividad de un país, por parte de los entes públicos dicha competitividad se alcanza por medio de la creación de marcos regulatorios que preparen las condiciones necesarias para el desarrollo de competitividad, de igual forma es necesario llevar a cabo un análisis de los factores que inciden en la competitividad mexicana para originar constructivismo en las áreas de oportunidad y con ello explicar la desigualdad en las ventajas competitivas.

Para Fuentes y Véliz (2017) la competitividad a nivel microeconómico se puede entender que la competitividad es cuando las empresas miden su capacidad para competir en el mercado, logrando conseguir sus beneficios económicos y expandirse; en lo macro se entiende por competitividad al nivel de capacidad que tiene un país por competir a niveles de mercado abierto, producción de bienes y servicios por otras economías que hacen crecer la renta nacional.

Por consiguiente es de suma importancia el imperativo acoplamiento de México hacia las tendencias globales y coyunturas políticas internacionales, como lo fue la época del proteccionismo estadounidense (2017-2021), ya que como bien menciona López et al. (2019) las economías emergentes como México, se caracterizan por un ambiente cambiante que obliga a las empresas, de todos los tamaños y sectores, a implementar estrategias que les permitan mantenerse en el mercado a través de la detección de sus fortalezas, debilidades, oportunidades y amenazas a través de un proceso de planeación estratégica, que les asegure el logro de objetivos y la sustentabilidad de la 
organización, a final de cuentas "Hay algo que tienen en común la productividad, la competitividad y la innovación. Cada uno de estos tres conceptos es considerado como clave para el crecimiento económico" (Meller, 2019)

La presente investigación tiene como objetivo identificar los factores que inciden en la competitividad de las empresas. Para identificar los factores se realizó una búsqueda exhaustiva de documentos científicos, de esta manera se creó un marco teórico/literario que estructura las variables determinantes de la competitividad.

\section{FUNDAMENTACIÓN TEÓRICA}

Como resultado de la búsqueda exhaustiva en la literatura científica para la elaboración de la presente investigación se encontraron los escritos de Molina (2010), Prado (2014), Ordóñez (2015) y Cuevas (2016) que fueron realizados en México y España.

La primera investigación de Molina (2010) precisó como propósito determinar y analizar los factores que explican la competitividad (evaluada en términos de productividad laboral) de la industria manufacturera en los municipios de la frontera norte de México, en los años 1994, 1999 y 2004, este tuvo como resultado a partir de la realización de un modelo panel de efectos fijos aplicado para la industria manufacturera concluyendo que la industria manufacturera ha presentado serias dificultades para sobrellevar las condiciones del mercado globalizado, ocasionando el debilitamiento de la cadena productiva y un cambio en la dinámica regional del país, debido a que en la industria manufacturera el tamaño medio de los establecimientos es diverso, lo que genera como consecuencia que esto no sea una condición de la productividad laboral, no obstante, no lo excluye de ser es un determinante significativo.

Además, dicha investigación también tuvo como resultado que la inversión en activos fijos repercute negativamente, aunque en una proporción mínima, sobre la productividad laboral debido a que los datos mostraron que la formación bruta de capital fijo en la frontera norte presentó nulo incremento en términos netos de 1994 a comparación del 2004.

El segundo estudio de Prado (2014) investigó las políticas públicas implementadas en el marco del Tratado de Libre Comercio de América del Norte (TLCAN) enfocándose en el sector automotriz realizando un estudio evaluativo de diseño longitudinal, la misma utilizó estadísticas de México de los resultados obtenidos durante tres sexenios Presidenciales (Ernesto Zedillo Ponce de León, Vicente Fox Quezada y Felipe Caderón Hinojosa) abarcando desde 1994 hasta 2012. Los resultados evidencian que las políticas públicas tienen impacto en la competitividad e innovación tecnológica, lo que resulta en fluidez para la inversión en investigación y desarrollo, en tecnología y dirección administrativa, según lo manifestado en los resultados estadísticos resulta en consecuencia que la remuneración económica laboral goce de un impacto positivo, por lo cual las políticas públicas fueron un factor determinante para apoyar impulsar e incentivar la competitividad en el sector automotriz en el marco del TLCAN.

La tercera investigación de Ordóñez (2015) estimó como objetivo principal identificar de forma empírica, los principales factores de la relación entre competitividad, bienestar y desarrollo humano en las entidades del norte de México para generar un marco de referencia que permita a las instituciones públicas considerar los aspectos fundamentales al definir los parámetros para propiciar el desarrollo humano. El autor utilizó el método estadístico a través del análisis de componentes principales y la elaboración de modelos de regresión. Los resultados arrojados presentaron que las principales variables identificadas para medir la competitividad y bienestar en las empresas fueron: por el lado del bienestar, se identificó la pobreza, la desigualdad y la cobertura social, mientras que por el lado competitivo se identificó: la productividad, recaudación de la banca comercial (ahorro) e inversión extranjera, por último se reconoció que existe una relación significativa entre el desarrollo humano y la competitividad, de tal manera que las entidades federativas mexicanas más competitivas presentan mayores niveles de desarrollo humano. 
Por último el autor agrega que en base a los resultados obtenidos, el Estado debe de buscar el desarrollo del bienestar por encima de la competitividad, para este la competitividad debe ser vista como un efecto de la globalización que tiene como fin la consolidación de un mercado estable, eficiente, de calidad y sobre todo de que genere certidumbre, calidad, y todo aquello sería protegido por instituciones públicas que regulen, estabilice y legitimen al mercado para impulsar el desarrollo, de tal manera que a mejores políticas sociales y regulación correcta local del mercado se podrá alcanzar altos índices de competitividad, dejando consigo una reflexión final en la cual argumenta que a pesar de vivir en un mundo globalizado está en su matriz depende mucho de las condiciones sociales y económicas locales.

La cuarta y última investigación de Cuevas (2016) tuvo como fin realizar un análisis acerca de la incidencia de la innovación y el capital intelectual en la competitividad de las PYMES (pequeñas y medianas empresas) manufactureras en Aguascalientes, utilizando como instrumento el análisis de regresión lineal, dicho instrumento fue aplicado a los gerentes de 150 PYMES. Como resultado se obtuvo que tanto la innovación y el capital intelectual influyen de manera positiva y significativa en la competitividad de las PYMES por lo cual se sugiere que los gerentes deben poner especial énfasis en la supervisión y aplicación de estas, recomendando mejoras continuas a todos aquellos elementos relacionados con su sistema de producción y administrativo además de invertir en capital intelectual con el propósito de crecer y eficientizar las acciones operativas dentro de las empresas.

\section{METODOLOGÍA O DISEÑO DE INVESTIGACIÓN}

La presente investigación es de índole documental y exploratorio abarcando la recolección de literatura científica para realizar dicho estudio. El diseño de esta investigacion es de caracter documental y exploratorio siendo no experimental y descriptivo, debido a que se pretende analizar, observar y describir la información sin algún tipo de práctica o técnica que altere los resultados dados de manera natural, según Hernandez et al. (2014) realizar dicha acción nos puede ayudar a entender el fenómeno central de estudio, y le sirve al investigador para conocer los antecedentes de un ambiente, así como las vivencias o situaciones que se producen en él y su funcionamiento cotidiano y anormal debido a que los resultados pretenden ser generales para que en el futuro puedan ser utilizados como punto de partida para futuras investigaciones con objetivos más concretos de caracter cuantitativo.

Para alcanzar el objetivo del presente estudio, se aplicó la técnica cualitativa a través de la revisión y recopilación consistente de literatura científica acerca de investigadores que realizaron estudios de campos en distintos países, con el fin de obtener información concreta y definir los principales factores que inciden en la competitividad de las empresas.

\section{RESULTADOS}

Por lo que respecta a los resultados obtenidos se encontró que los factores que inciden en la competitividad derivan en ocho variables, las cuales fueron: 1. Innovación tecnológica, 2. Factores humanos, 3. Recursos y capacidades, 4. Desarrollo en la investigación, 5. Entorno, 6. Infraestructura física, 7. Proceso productivo y 8. Materiales y energía.

\section{Innovación tecnológica}

En base a lo referido por la OCDE (Organización de Cooperación y Desarrollo Económico, 1996) la define como la primera aplicación de la ciencia y la tecnología en una nueva dirección que da como resultado un éxito comercial seguro, en palabras de Cuevas (2016) tanto la innovación 
como la tecnología son consideradas por las empresas como los mecanismos más importantes por el impacto que tienen en la competitividad empresarial.

Morales (2021) Argumenta que mediante la innovación, se demostró cómo se pueden crear ideas para nuevos bienes y servicios, producidos por nuevas tecnologías, en la economía de mercado. También se demostró cómo este cambio tecnológico endógeno puede moldear el crecimiento y qué políticas son necesarias para que los procesos funcionen bien.

El acceso a la innovación tecnológica aunado a las brechas digitales tanto en capacitación como en la educación en ella, representa uno de los mayores retos para México. El TLCAN permitió a México crear productos de una mejor calidad debido a que la demanda creada provoca que haya más producción industrial, la cual termina siendo exportada en su mayoría a los Estados Unidos, quien es el principal mercado meta de las industrias, sin embargo, para poder mantener esta ventaja es necesario una mejora continua de inversión en ciencia y tecnología por parte de las empresas mexicanas además del aprovechamiento de la innovación tecnológica e inversión que proveen las empresas extranjeras que se establecen en México.

\section{Factores humanos}

Los factores humanos están relacionados en gran proporción a la productividad, apunta Cequea et al (2011) en este sentido se constituye una naturaleza en la que influyen características y comportamientos del individuo, dicha influencia implica procesos psicológicos y psicosociales que repercuten en la productividad y competitividad de las empresas.

Con lo anterior expuesto, el resultado de la armonía y articulación entre la tecnología, los recursos humanos, la organización y los sistemas gerenciados o administrados por las personas, refiere Tolentino (2004) que la productividad se manifestará siempre que se consiga la combinación óptima o equilibrada de los factores humanos, Delgadillo (2003) expone que a mayor optimización de los factores humanos, en consecuencia mayor será la productividad, lo que a su vez mayor generación de eficiencia y competitividad.

Dicho de otra manera, para que una economía logre ser competitiva de manera sólida es necesario que esta sea altamente productiva esto es importante "porque determina la remuneración de los trabajadores e inversores en concepto de salarios, utilidades y rendimiento de capital, además la productividad marca el límite a las posibilidades del recurso humano de mejorar su calidad de vida" (Huerta 2017).

En particular los factores humanos desde la perspectiva de los recursos humanos desempeñan un rol clave en la competitividad de las empresas mediante la eficiencia que se obtiene al desarrollar comunicación continua con cada uno de los elementos en los diferentes niveles; al renovarse continuamente, innovando en los procedimientos, permitiéndose evolucionar y evitando la obsolescencia a través de la excelencia.

\section{Desarrollo de la investigación}

Navas (2017) menciona que en un sistema competitivo, la investigación, el desarrollo y la innovación son factores que inciden en una forma directamente proporcional en el crecimiento de las empresas e influyen positivamente en la creación de valor.

La investigación de Rickne et al. (2013) colocan a la universidad en el papel principal del proceso de innovación y producto de conocimiento, aunque en algunas situaciones el gobierno asume el papel principal o dominante en el desarrollo de proyectos y proporciona los recursos para la nueva iniciativa y que esta sea aplicable al sector productivo en las empresas.

Por lo tanto se estima que el presente factor debe de ser desarrollado por tres actores para poder alcanzar niveles óptimos, estos son la triple hélice del desarrollo (THD): las universidades, las cuales son fundamentales para empoderar a futuras generaciones por medio del conocimiento debido a que son la fuente de capital humano que estará presente en todos los niveles de las empresas. 
El segundo actor son las empresas que por medio de inversión en capital humano y económico tanto en áreas clave, podrán impulsar su competitividad, creando y produciendo productos y servicios innovadores de acuerdo a la demanda y las necesidades actuales

El tercer actor es el sector público quien prepara las condiciones por medio de políticas públicas y el marco legal, mismos fungen como mecanismos para crear las condiciones necesarias para el desarrollo, afirma Malaver (1999) la necesidad de impulsar las transformaciones en las organizaciones reclama mayores esfuerzos de investigación de la realidad empresarial, incrementar las demandas por nuevos conocimientos y prácticas empresariales en el desarrollo de investigación ayudará a orientar a las empresas a mejorar su competitividad.

Dichos actores deben de colaborar estrechamente para que un país pueda ser competitivo tal es el caso de Corea del Sur, son un ejemplo de éxito del engranaje de la triple hélice del desarrollo (THD), entre entes públicos, académicos y empresariales en lo referente al desarrollo de la investigación aplicada en la era de la globalización, esto los ha vuelto productivos y competitivos.

\section{Proceso productivo}

Porter (1990) apunta que el concepto más relevante referente a la competitividad es la productividad, la cual tiene como principal objetivo el crecimiento de las empresas e incrementar el estándar de vida de los elementos que la conforman por medio de procesos de producción, según Donelly et al. (1994) la función de producción en una organización de negocios se ocupa específicamente de la actividad de producción de artículos, es decir, el diseño, la implantación, la operación y el control del personal, materiales, equipos, capital e información para lograr objetivos específicos de producción.

Rodríguez et al. (2002) menciona que el proceso productivo, se caracteriza por la combinación equilibrada de una serie de complejas actividades y elementos, que comprenden el diseño del producto, la selección del sistema productivo y de la tecnología, la planificación de la capacidad, la ubicación y distribución de las instalaciones y el diseño de los procesos para la obtención del resultado deseado mencionan sin embargo; Chase et al. (2000) lo complementan añadiendo que además del diseño conceptual del producto hay que tomar en cuenta el mercado objetivo, el nivel deseado de desempeño, los requerimientos de inversión y el impacto financiero.

De esta forma, las PYMES podrían actuar como agentes que fomenten el cambio estructural, ayudando a la creación y difusión de innovaciones y el desarrollo de nuevos mercados indican Altenburg et al. (2006). Por consiguiente, la entrada de nuevas empresas podría aumentar la competencia, incorporar nuevos modelos de negocios que desafían los tradicionales generando una turbulencia saludable que conlleva a la búsqueda constante de un uso más productivo de los recursos y contribuye al aumento de la productividad agregada de una economía.

\section{Recursos y capacidades}

Corona (2005) indica que muchas industrias carecen de capital de riesgo, es decir no designan recursos a actividades de innovación, por ejemplo, por temor de perder esos recursos en el ámbito de la integración económica regional, trae consigo que el campo donde se desarrollen las empresas sea fuertemente competitivo comparándolo a cómo se desarrollaba en el pasado, optando por valerse de sus propios recursos y capacidades para conseguir gestionarse de manera eficiente y mantenerse vigentes. La competencia desmesurada es consecuencia del proceso de globalización, lo que conlleva a la búsqueda de estrategias que les permitan sobrevivir y adaptarse al entorno continuamente cambiante, mediante la identificación de las capacidades generadoras de valor.

Cardona (2011) señala que los recursos y capacidades, han sido dos campos en los que tradicionalmente se agrupan los estudios de la estrategia, estos mismos se han convertido en un factor determinante para la producción y la competitividad, dada la complejidad y la acelerada turbulencia en la que se mueven los mercados, sin ellos las empresas difícilmente podrán maniobrar 
en los mercados, por consiguiente se ubica este factor en el campo de conocimiento, de la gestión y en la estrategia.

\section{Entorno}

El entorno donde se desarrollan las empresas, sea cual sea su rubro es fundamental para generar competitividad, en palabras de Baena et al. (2003) los resultados internos de las empresas dependen en gran medida de las características del entorno (endógeno-exogeno) en que se desarrollan y de la capacidad que tienen de asimilarlo, además de administrar eficientemente los recursos. Una mirada rápida al entorno actual de las empresas nos muestra que éste no es lineal y que no está conformado únicamente por variables cuantitativas fácilmente manipulables, sino cualitativas de igual forma, dicho de otra manera, el entorno se desarrolla y complementa en coyunturas entre factores endógenos y exógenos.

Por un lado, el entorno depende en amplia medida de variables cualitativas: recursos humanos, lazos de intereses económicos, poder, influencia, diferenciación estructural empresarial interna, cada una de ellas siguen un camino propio sin una esquematización general, lo que lleva a cada una a desarrollar en su núcleo un entorno distinto pero al mismo tiempo este repercute en su competitividad a nivel local como internacional.

Por otro lado, los elementos externos como: la inflación, fluctuación en tipos de cambio, el gobierno en turno, la burocracia y las tasas de interés, serán determinantes en el desarrollo de la empresa y la competitividad de la misma, razón por la cual, en este caso, son los actores gubernamentales los que deben de generar certidumbre para las empresas, tanto extranjeras, nacionales y en partículas las PYMES.

\section{Infraestructura física}

Los bienes de capital que en forma de equipamiento, facilitan la producción mediante el transporte de energía, se entienden como infraestructura física, indica Buhr (2009) contribuyen a la producción de bienes y servicios necesarios para satisfacer los requisitos básicos de los agentes económicos. En última instancia la infraestructura busca colaborar con el sector social y productivo para que la producción en masa sea económicamente factible y accesible.

Sin embargo, se debe reiterar que crear infraestructura no es sinónimo de competitividad, para que esta sea competente, primero se debe hacer un análisis para saber qué tipo de infraestructura es la necesaria, esta puede ser de tipo social, la cual tiene como objetivo mejorar las condiciones de la comunidad, desde la creación de puentes, caminos o tuberías o de inversión, para permitir celeridad de conexión entre un punto del territorio u otro al final dependerá de la valoración y análisis estratégico, que puede ser realizado región por región.

\section{Materiales y energía}

En la era de globalización la tendencia apunta a la optimización de materiales y energía para lograr un equilibrio sustentable con el medio ambiente, en teoría una empresa competitiva debe de pensar de forma sustentable en cuanto al uso de materiales y energía, siendo un objetivo de ámbito social de producción, como resultado la correcta eficiencia de recursos tendrá como consecuencia directa un mayor aumento en producción y calidad, sin perjudicar presentes y futuras generaciones.

Reyes et al (2014) afirma que dicho de otra forma un beneficio para las empresas que se dedican a la elaboración de productos de consumo o para la industria, es que se pueden aprovechar los desechos para no generar pérdidas, al contrario obtener ingresos.

Piñeiro et al. (2001) indica que las empresas deben comenzar a considerar el uso de energías limpias y renovables como prioridad, debido a que las energías renovables proponen una alternativa válida desde el punto de vista social y económico con la visión de lograr un desarrollo sostenible. Coello (2019) propone que el hecho de que las energías renovables se identifiquen con rentabilidad a futuro en tanto fuente de ventaja competitiva no es sino un aliciente para liderar este proceso de transformación. 
Los factores mencionados y descritos en el presente artículo fueron los identificados en las bases de datos científicas, como los principales que inciden sobre la competitividad en las empresas. A continuación se recopila un marco teórico /literario que recopila todas aquellas investigaciones que han estudiado las variables con sus objetos de estudio y el lugar donde estas fueron llevadas a cabo (Ver tabla I).

Tabla I.

Factores que inciden en la competitividad de las empresas

\begin{tabular}{|c|c|c|c|}
\hline Factor & Objetos de estudio & Investigaciones & Lugar \\
\hline $\begin{array}{l}\text { Innovación } \\
\text { tecnológica }\end{array}$ & $\begin{array}{l}\text {-Perfeccionamiento de la calidad. } \\
\text {-Introducción de nuevos métodos de comercialización. } \\
\text {-Acceso a las tecnologías de información. } \\
\text {-Actividades de investigación y desarrollo (I\&D). } \\
\text {-Departamento de producción. } \\
\text {-Los clientes. }\end{array}$ & $\begin{array}{l}\text { Peñaloza (2007), Prokopenko (1989), } \\
\text { López et al. (2005), Álvarez et al. } \\
\text { (2003), Hidalgo (1999), Ghezán, Acuña } \\
\text { et al (2003) Moreno (2014), Porter } \\
\text { (1990), Witker (2011), Etzkowitz et al } \\
\text { (2000), Corona (2002), Prado (2014), } \\
\text { Jiménez-García et al. (2011), Farfán et } \\
\text { al. (2012) }\end{array}$ & $\begin{array}{l}\text { España, } \\
\text { Venezuela, } \\
\text { Suiza, Uruguay, } \\
\text { México y } \\
\text { Estados Unidos }\end{array}$ \\
\hline $\begin{array}{l}\text { Factores } \\
\text { humanos }\end{array}$ & $\begin{array}{l}\text {-Motivación } \\
\text {-Satisfacción laboral } \\
\text {-Competencias } \\
\text {-Formación y desarrollo } \\
\text {-Participación } \\
\text { Trabajo en equipo /Cohesión } \\
\text {-Clima organizacional / Manejo del conflicto } \\
\text {-Cultura organizacional } \\
\text {-Liderazgo }\end{array}$ & $\begin{array}{l}\text { Cequea et al. (2011), Barney (1986), } \\
\text { Miles et al. (1978), Samaniego (1998), } \\
\text { Kemppila et al. (2003), Tolentino, } \\
\text { (2004), Saari et al. (2004) Jones et al. } \\
\text { (2006), Antikainen et al. (2006), } \\
\text { Quijano (2006), Kemppila et al. (2003) } \\
\text { Fernández-Ríos et al. (1997), Jones } \\
\text { (1995) Locke et al. (1981), Mowday et } \\
\text { al. (1979), Estrada et al. (2007). }\end{array}$ & $\begin{array}{l}\text { Suiza, } \\
\text { Venezuela, } \\
\text { Estados Unidos, } \\
\text { España, Reino } \\
\text { Unido y México }\end{array}$ \\
\hline $\begin{array}{l}\text { Recursos y } \\
\text { capacidades }\end{array}$ & $\begin{array}{l}\text { A Nivel gerencial: } \\
\text {-Evaluación de las técnicas de la administración financiera. } \\
\text {-Sistema de control formales } \\
\text {-Centros de costos } \\
\text {-Rentabilidad } \\
\text {-Valuación de los activos y del desempeño de } \\
\text { colaboradores } \\
\text {-Reporte de ingresos y egresos } \\
\text {-Habilidad para la formación de equipos } \\
\text {-Conciliación de los sistemas de control } \\
\text {-Empoderamiento y estilos de dirección } \\
\text {-Planeación y políticas estratégicas } \\
\text {-Resistencia al cambio, ansiedad } \\
\text {-Comprensión y compromiso. } \\
\text { A Nivel funcional: } \\
\text {-Relación con los clientes } \\
\text {-Conocimiento y calidad del servicio o producto } \\
\text {-Garantías ofrecidas } \\
\text {-Índice de satisfacción, } \\
\text {-Monto de comprar } \\
\text { A nivel gubernamental: } \\
\text {-Entorno internacional } \\
\text {-Promoción comercial } \\
\text {-Cultural y de imagen } \\
\text {-Diplomacia Pública }\end{array}$ & $\begin{array}{l}\text { Ynzunza et al. (2008), De Sarbo et al. } \\
\text { (2003), Eisenhardt et al. (2000), } \\
\text { Hernández et al. (2014), Chandler } \\
\text { (1990), Collis (1994), Zhao et al. } \\
\text { (2001), Nelson et al. (1982), Birrchall } \\
\text { et al. (2002), Castro (2010), González } \\
\text { et al. (2007), Schiavon et al. (2019), } \\
\text { Lerner et al. (2002), Ynzunza et al. } \\
\text { (2013), Prokopenko (1989). }\end{array}$ & $\begin{array}{l}\text { Suiza, México, } \\
\text { Estados Unidos } \\
\text { y Costa Rica }\end{array}$ \\
\hline
\end{tabular}

-La academia

-Programas universitarios de emprendimiento

-Vinculación entre las univesidades, las empresas y el

Desarrollo en

la

investigación

-Investigación

-Sistema por competencias

-Programas nacionales de estímulos a la investigación al sector productivo. al. $(2012)$ Fernández-Ríos (1995) Locke et al. (1981), Mowday et al. (1979), Estrada et al. (2007).

Ynzunza et al. (2008), De Sarbo et al. (2003), Eisenhardt et al. (2000), Cornández al. (2014), Chandler et al. (2007), Schiavon et al. (2019) Lerner et al. (2002), Ynzunza et al. (2013), Prokopenko (1989).

Rickne et al. (2013), Navas (2017), Hernández et al. (2017), Bautista (2015), Kinner et al. (2008), McGuinness (2008), Mota et al. (2012), Rivera et al. (2011), Rivera et al. (2013), Saavedra (2009)
Suiza, México, Estados Unidos
-La inflación

-El déficit presupuestario

-Los tipos de cambio

-La deuda externa

Entorno empresarial
-Gobierno en turno

-Producto interno bruto
Westreicher (2020), Thompson et al. (2012), Arano et al. (2013), Esser et al. (1994), Smallbone et al. (2001), Olawale et al. (2010), Blecker (2009), Ros (2004), Avendaño et al. (2011) Hopfenbeck (1993), Chamorro, (2002),
México, España, Alemania, 
-Reducir la burocracia

-Tasas de interes

-Las prácticas de los organismos estatales

-Los reglamentos (como las políticas de control de precios,

Infraestructura

física

-El transporte y las comunicaciones;

-Las medidas y los incentivos fiscales (tipos de interés,

aranceles aduaneros, impuestos)

-Esquemas de inversión y modelos de financiamiento

-Planeación a largo plazo,

-Análisis en las licitaciones

-Uso deficiente de los recursos y riesgos desbalanceados entre contratantes y contratistas.

$\begin{array}{ll} & \text {-Materia prima } \\ & \text {-Mano de obra } \\ & \text {-Método de trabajo } \\ & \text {-Máquinas } \\ \text { Proceso } & \text {-Medio ambiente } \\ \text { Productivo } & \text {-Medición } \\ & \text {-Diseño del producto, } \\ & \text {-La planificación de la capacidad } \\ & \text {-La ubicación y distribución de las instalaciones } \\ & \text {-El diseño de los procesos }\end{array}$

-Rendimiento del material: Producción de productos útiles o de energía por unidad de materiales utilizados.

-Uso y control de desechos y sobras

-Perfeccionamiento de los materiales mediante la elaboración inicial para mejorar la utilización en el proceso principal.

-Mejoramiento del índice de rotación de las existencias energía para liberar fondos vinculados a las existencias con el fin de destinarlos a usos más productivos.

-Mejoramiento de la gestión de existencias para evitar que

se mantengan reservas excesivas

-Uso de energías renovables.

Coddington (1993), Davis (1993), Croacia,

Hartmann et al. (2003) Sudáfrica.

Sylos (1993), Schumpeter (1938), Prokopenko (1989), Aschauer (1990), Cerón (2018), Nijkamp et al. (1999), Keynes (1943), Taaffe et al. (1963), Rostow (1967), García (2002), Maza et al. (2012), García (2019) Benzaquen (2010), Barbero (2011), Perrotti et al. (2011).

Suiza, México, España, Estados

Unidos y

Dinamarca
Porter (1990), Cann (2016), Altenburg et al. (2006), Rodríguez Medina, et al. (2002), Donelly et al. (1994), Ferraro et al. (2010), Chase et al. (2000), Buffa et al. (1992), Hansen et al. (1996).
Estados Unidos, Suiza, México, Latinoamérica.
Miño-Cascante (2015) Polimeni et al. (1994), Prokopenko (1989), Vargas (2020), Reyes et al. (2014), Piñeiro et al. (2001), Coello (2019)
Cuba, México, Suiza y España.

Fuente Elaboración propia.

\section{CONCLUSIONES}

Las variables expuestas en el presente artículo, representan numerosos constructores soportados por investigaciones concebidas en diversas partes del mundo, en donde la definición de cada una de ellas converge, sin embargo, se pueden interpretar de diferentes maneras, en la teoría y en la práctica.

\subsection{De la comprobacion cualitativa.}

Desde una perspectiva amplia de la problemática sobre los factores que inciden en la competitividad en las empresas, no existe un factor cualitativo o cuantitativo preponderante permanentemente, sino una simbiosis natural en la que predomina uno del otro dependiendo la coyuntura manifestada, por ello el experto debe ser lo suficientemente hábil para identificar los factores con áreas de oportunidad expuestas y actuar en medida para alcanzar el grado de competitividad anhelada.

Dicho de otra manera, el entorno de las empresas nos muestra que no actúa de manera "lineal" y que no está conformado principalmente por variables cuantitativas que puedan manipularse de manera sencilla y que además dependen en gran proporción a factores cualitativos que en gran dimensión siguen un camino propio sin una esquematización general, lo que conduce a 
cada una a coexistir en su núcleo un entorno distinto, pero que al mismo tiempo este concierne en su competitividad a nivel tanto local como internacional.

En síntesis, para hacer frente a la híper competitividad, las empresas deben ir en tendencia con la globalización, en una nueva dirección, apoyándose de los factores que inciden en la competitividad tales como la ciencia y la tecnología, además de la innovación tecnológica, lo cual dará como resultado un éxito comercial, debido a que son consideradas por diversos investigadores como los instrumentos más importantes en la competitividad empresarial.

\subsection{De las limitaciones del estudio}

Para finalizar con las aclaraciones de esta investigación, es menester puntualizar las limitaciones que se presentaron a lo largo de su desarrollo. La limitante principal del presente estudio es la escasa literatura cientifica a nivel nacional (México) existente en este tema, la cual tuvo que buscar fuentes internacionales para ser elaborada.

\subsection{De las Recomendaciones}

Esta investigación exhorta a la comunidad científica interesada en esta problemática a que se repliquen estudios más amplios en donde aporten técnicas cualitativas y cuantitativas para respaldar la fiabilidad de los factores recolectados en la literatura científica, de esta manera se tendran resultados con fiabilidad y representacion muestral de la informacion que aporten herramientas que puedan ser aplicadas de alguna manera a la practica de los negocios internacionales o el comercio exterior para un impulso y mejoramiento de las empresas nacionales en su proceso de integracion al mundo globalizado.

\section{REFERENCIAS}

Altenburg, T., \& Eckhardt, U. (2006). Productivity Enhancement and Equitable Development: Challenges for SME Development. Unidos.

Álvarez, V., y Rodríguez, D. (2003). Del Sector Ciencia y Tecnología a la Sociedad del Conocimiento, Fundación Centro Gumilla.

Antikainen, R., \& Lbnnqvist, A. (2006). Knowledge Work Productivity Assessment. Institute of Industrial Management.

Arano Chávez, R., Cano Flores, M., y Olivera Gómez, D. (2013). La importancia del entorno general en las empresas. Investigaciones y Estudios Superiores de las Ciencias Administrativas, pp. 62-65. Obtenido de: https://www.uv.mx/iiesca/files/2013/04/06CA201202.pdf

Aschauer, D. (1990). Why is infrastructure important? Federal Reserve Bank of Boston Conference Series, pp. 21-68. Obtenido de: https://econpapers.repec.org/article/fipfedbcp/y_3a1990_3ap_3a21-68_3an_3a34.html

Avendaño, V., y Vázquez, J. (2011). Inestabilidad financiera y política monetaria en México, 1990-2008. Investigación Económica, 7 (275), pp. 63-92. Obtenido de: http://www.scielo.org.mx/pdf/ineco/v70n275/v70n275a3.pdf.

Baena, E., Jairo, J., y Montoya, O. (2003). El entorno empresarial y la teoría de las cinco fuerzas competitivas. Scientia et Technica, 3 (23), pp. 61-66. Obtenido de: https://dialnet.unirioja.es/servlet/articulo?codigo=4845158

Barbero, J. (2011). La infraestructura en el desarrollo integral de América diagnóstico estratégico y propuestas para una Agenda. CAF.

Barney, J. (1986). Strategic factors: markets, expectations, luck, and business strategy. Management Science, 32(10), 1231-1241. Obtenido de: https://www.jstor.org/stable/2631697?seq=1

Bautista, E. (2015). La importancia de la vinculación universidad-empresa-gobierno. Revista Iberoamericana para la Investigación y el Desarrollo Educativo, 5(10). Doi: 10.23913/ride.v5i9.106 
Benzaquen, J. (2010). Un índice regional de competitividad para un país. CEPAL, pp. 69-86. Obtenido de : https://repositorio.cepal.org/bitstream/handle/11362/11417/102069086_es.pdf?sequence=1\&isAllowed=y

Birrchall, D., \& Tovstiga, G. (2002). Assessing the firm's strategic knowledge portfolio: A framework and methodology. International Journal of Technology Management.

Blecker, R. (2009). External Shocks, Structural Change, and Economic Growth in Mexico (1979-2007). World Development, 37(7), pp. 1274-1284. Obtenido de: DOI: 10.1016/j.worlddev.2008.10.004.

Buffa, E. S., y Sarin, R. K. (1992). Administración de la Producción y de las Operaciones. Limusa Grupo Noriega.

Buhr, W. (2009). Infrastructure of the Market Economy. Volkswirtschaftliche Diskussionsbeitrage, 132 (40), pp. 1-74. Doi: 10.21670/ref.2012.25.a03

Cann, O. (2016a). ¿Qué es la competitividad? World Economic Forum.

Cann, O. (12 de Octubre de 2016). Weforum. Obtenido de Weforum: https://es.weforum.org/agenda/2016/10/que-es-lacompetitividad/

Cardona, R. A. (2011). Estrategia basada en los recursos y capacidades. criterios de evaluación y el proceso de desarrollo. Revista Electrónica Forum, 4, pp. 113-147. Obtenido de: https://core.ac.uk/download/pdf/290653867.pdf

Castro. E. (2010). Las estrategias competitivas y su importancia en la buena gestión de las empresas. Ciencias Económicas, 28 (1) pp. 247-276. Obtenido de: https://dialnet.unirioja.es/servlet/articulo?codigo=3406855

Cequea, M., y Núñez, M. (2011). Factores humanos y su influencia en la productividad. Revista Venezolana de Gerencia, 16 (53), pp. 116-137. Obtenido de: https://www.redalyc.org/pdf/290/29018414007.pdf

Cerón, M. (2018). La infraestructura y su relación con el desarrollo económico de un país: Caso de América Latina y el Caribe.Univevrsidad Nacional Autónoma de México.

Chamorro, A. (2002). La gestión ambiental como ventaja competitiva. Obtenido de 5campus.org: https://ciberconta.unizar.es/leccion/gestmed/inicio.html

Chandler, A. (1990). Strategy and structure: Chapters in the history of the industrial enterprise. Cambridge Mass.

Chase, R., y Aquilano, N. (1994). Dirección y Administración de la Producción y de las Operaciones. Addison Wesley Ibero American.

Chase, R., Aquilano, N., y Jacobs, R. (2000). Administración de Producción y Operaciones. McGraw-Hill Interamericana.

Coddington, W. (1993). Environmental Marketing. McGraw-Hill.

Coello, M. (2019). La ventaja competitiva de las energías renovables. Comillas Universidad Pontifica.

Collis, D. (1994). Research note: How valuable are organizational capabilities? Strategic Management Journal, pp. 143152.Doi: $10.1002 / \mathrm{smj} .4250150910$

Corona, L. (2005). México: el reto de crear ambientes regionales de innovación. Centro de Investigación y Docencia Económicas.

Cuevas, V. (2016). La influencia de la innovación y la tecnología en la competitividad de las PYMES manufactureras del Estado de Aguascalientes. Universidad Autónoma de Aguascalientes.

Davis, J. J. (1993). Strategies for Environmental Advertising. Journal of Consumer Marketing, 10(2), pp. 19-36. Doi: $10.1108 / 07363769310039102$

De Sarbo, W., Benedetto, A., Jedidi, K., \& Song, M. (2003). A Constrained Finited Mixture Structural Equation Methodology for Empirically Deriving Strategic Types. Pennsylvania: Institute for the Study of Business Markets.

Delgadillo, L. (2003). Modelo para evaluar la productividad en micro, pequeñas y medianas empresas de la cadena productiva de la electrónica, la informática y las telecomunicaciones en el estado de Jalisco, México. $27^{\circ}$ Congreso Nacional de Estadística e Investigación Operativa. Universidad de Guadalajara.

Donelly, J. H., Gibson, J. L., \& Ivancevich, J. M. (1994). Dirección y Administración de Empresas. Addison-Wesley Iberoamerican.

Eisenhardt, K., \& Martin, J. (2000). Dynamic Capabilities: What are they? Strategic Management Journal, pp. 11051121. Doi: 10.1002/1097-0266(200010/11)21:10/11<1105::AID-SMJ133>3.0.CO;2-E 
Esser, K., Wolfang, H., y Meyer-Stamer, J. (1994). Competitividad sistémica. Competitividad internacional de las empresas y políticas requeridas. Instituto Alemán de desarrollo.

Estrada, S., y Restrepo, L. (2007). Cómo influyen los factores de motivación en la satisfacción de los empleados dentro de una organización. Scientia et Technica, 1 (37) pp. 345-350. Doi: 10.22517/23447214.4089

Etzkowitz, H., \& Leydesforff, L. (2000). The dynamics of innovation: from National Systems and "Mode 2" to a Triple Helix of university-industry-government relations. Research Policy, 29(2), pp. 109-123. Doi: 10.1016/S00487333(99)00055-4.

Farfán, G., y Velázquez, R. (2012). Presupuesto y política exterior en México planeación estratégica, incrementalismo y discrecionalidad. Revista legislativa de estudios sociales y de opinión pública, 5 (9), pp. 39-101. Obtenido de : https://dialnet.unirioja.es/servlet/articulo?codigo $=4169371$

Fernández-Ríos, M., y Sánchez, J. (1997). Eficacia organizacional.Concepto, desarrollo y evaluación. Díaz de Santos.

Ferraro , C., y Stumpo, G. (2010). Políticas de apoyo a las pymes en América Latina Entre avances innovadores y desafios institucionales. CEPAL

Fuentes, E., \& Véliz, G. (2017). La competitividad Global. Revista Empresarial, ICE-FEE-UCSG, 41-47.

García, J. A. (2002). La red ferroviaria Andaluza: infraestructuras y modelo territorial. Cuadernos Geográficos, 32, pp. 97-123. Obtenido de : https://www.redalyc.org/pdf/171/17103205.pdf

Ghezán, G., Acuña, A., Viteri, M., y Demari, G. (9 al 11 de Julio de 2003). Comportamiento innovativo de PyMEs Agroalimentarias Argentinas. Estudio de Casos

González, E., y Ventura, J. (2007). Variedad estratégica y rentabilidad empresarial en la industria manufacturera. Economía Aplicada, 15 (43), pp. 71-94. Obtenido de : https://www.redalyc.org/articulo.oa?id=96915879003

Hansen, D., y Mowen, M. (1996). Administración de Costos. Contabilidad y Control. International Thomson.

Hartmann, P., Forcada Sainz, F. J., y Apaolaza Ibañez, V. (2003). Superando los límites medioambientales de la empresa:Un estudio experimental del efecto del posicionamiento. Universidad del País Vasco-Euskal Herriko Unibertsitatea, 4 (1), 83-95. http://www.ehu.eus/cuadernosdegestion/documentos/415.pdf

Hernández, N., Sánchez, Y., y Verástegui, J. (2014). Identificación de las capacidades empresariales como factor generador de la competitividad en el sector hotelero de Cd Victoria, México. Investigación administrativa, 43, (133) pp. 78-94. Obtenido de :

http://www.scielo.org.mx/scielo.php?pid=S244876782014000100078\&script=sci_abstract

Hernández, C., y Sánchez, S. (2017). La educación empresarial: un acercamiento desde los estudiantes universitarios en dos instituciones de educación superior. Innovación educativa, 17(75) pp. 81-102. Obtenido de: http://www.scielo.org.mx/pdf/ie/v17n75/1665-2673-ie-17-75-81.pdf

Hernández, R., Fernández, C., y Baptista, P. (2014). Metodología de la investigación: Roberto Hernández Sampieri, Carlos Fernández Collado y Pilar Baptista Lucio (6a. ed. --.). McGraw-Hill.

Hidalgo Nuchera, A. (1999). La gestión de la tecnología como factor estratégico de la competitividad industrial. Economía Industrial, 6 (330), pp. 43-54. Obtenido de :

https://www.mincotur.gob.es/Publicaciones/Publicacionesperiodicas/EconomiaIndustrial/RevistaEconomiaInd ustrial/330/08ahid.pdf

Hopfenbeck, W. (1993). Dirección y marketing ecológicos. Ediciones Deusto.

Huerta, A. (2017). Impacto de la política proteccionista de Estados Unidos en la economía mexicana. ECONOMIA UNAM, 14(42). Obtenido de : http://www.revistas.unam.mx/index.php/ecu/article/view/61740/54365

Huerta, E. (2017). Diario de Noticias. Obtenido de Diario de Noticias: https://ifuturo.org/por-que-es-importante-laproductividad/\#: :text=En\%20una\%20econom\%C3\%ADa\%20competitiva\%20la,salarios\%20y\%20rendimient os $\% 20 \mathrm{de} \% 20$ capital.\&text=Adem $\% \mathrm{C} 3 \% \mathrm{~A} 1 \mathrm{~s} \% 2 \mathrm{C} \% 201 \mathrm{a} \% 20 \mathrm{evoluci} \% \mathrm{C} 3 \% \mathrm{~B} 3 \mathrm{n} \% 20 \mathrm{de} \% 201 \mathrm{a}, \mathrm{mejorar} \% 20 \mathrm{el} \% 2$ Onivel\%20de\%20vida

Jiménez-García, C., López-Lira, N., Tomta, D., y Pacheco-Olvera, A. (2011). Competitividad de la economía mexicana, resultados en el periodo 1997-2007. Convergencia, 18 (56) pp. 215-238. Obtenido de: http://www.scielo.org.mx/pdf/conver/v18n56/v18n56a9.pdf

Jones, T. (1995). "Instrumental Stakeholder Theory: A Synthesis of Ethics and Economics. Academy of Management Review, 20(2), pp. 404-437. Doi.org/10.5465/amr.1995.9507312924 
Kemppila, S., \& Lbnnqvist, A. (2003). Subjective Productivity Measurement. The Journal of American Academy, 2 (2) pp. 531-537. Doi:10.1155/2008/680159

Keynes, J. (1943). Teoría General de la ocupación, el interés y el dinero. Fondo de Cultura Económica.

Kinner, T., y Taylor, J. (2008). Investigación de Mercados: Un enfoque aplicado.

Lerner, M., \& Almor, T. (2002). Relationships among Strategic Capabilities and the Performance of Women-Owned Small Ventures. Journal of Small Business Management, 40 (2), pp. 109-125. Doi: 10.1111/1540-627X.00044

Locke, E. A., Shaw, K. N., Saari, L. M., \& Latham, G. (1981). Goal Setting and Task Performance: 1969-1980. Psychological Bulletin, 90, pp. 125-152. Obtenido de : http://garfield.library.upenn.edu/classics1992/A1992JF73700001.pdf

López, N., Montes, J., Vázquez, C., y Prieto, J. (2005). Innovación y Competitividad:implicaciones para la gestión de la innovación. Revista Tribuna de Debate.

López, M., Zalthen, L., \& Peraza, L. (2019). La planeación estratégica y la competitividad del sector restaurantero en ciudad del Carmen, Campeche, México. Revista de Investigación Latinoamericana en Competitividad Organizacional, 1-15.

Malaver, F. (1999). La investigación en gestión empresarial. Academia. Revista Latinoamericana de Administración, 23, pp. 62-77. Obtenido de : https://www.redalyc.org/articulo.oa?id=71602306

Maldonado, H. H., \& Ortiz, G. D. (2021). Retrospectiva comercial mexicana: del TLCAN al T-MEC. Sapienza: International Journal of Interdisciplinary Studies, 2 (1), PP. 370-384. DOI: 10.51798/sijis.v2i1.48

McGuinness, J. A. (2008). Globally competitive, Locally Engaged: The Case of Kentucky. Higher Education Management and Policy, 20 (2), pp. 1-16. Doi: 10.1787/hemp-v20-art13-en

Medina, J. E. (2007). Modelo Integral de Productividad. Fondo de Publicaciones.

Meller, P. (2019). Productividad, competitividad e innovación: perspectiva conceptual. Cieplan, 1-59.

Miles, R., \& Snow, C. (1978). Organizational Strategy, Structure, and Process. Mc Graw Hill.

Miño-Cascante, G., Saumell-Fonseca, E., Toledo-Borrego, A., Roldan-Ruenes, A., \& Moreno-García, R. (2015). Planeación de requerimientos de materiales por el sistema MRP.Caso Laboratorio. Cuba. Tecnología Química, 35 (2), pp. 248-260. Obtenido de: https://www.redalyc.org/pdf/4455/445543787008.pdf

Molina C. (2010). Análisis de los determinantes de la competitividad de la industria manufacturera en la frontera norte: 1994, 1999 y 2004. El Colegio de la Frontera Norte.

Morales, G. (2021). La innovación tecnológica: creando competitividad en las empresas desarrolladoras de software. Podium (39), 139-154.

Moreno, H. (2014). La innovación tecnológica como herramienta para el desarrollo de la competitividad en las pymes. Revista Iberoamericana de Contaduría, Economía y Administración, 2(3), pp. 1-11. Obtenido de: https://www.ricea.org.mx/index.php/ricea/article/view/15

Mota, Q. A., y de Ibarrola, M. (2012). Las competencias como referentes curriculares: el proceso de traducción de lo laboral a la formación en las Universidades Tecnológicas. Revista de la educación superior, 41 (164) pp. 3555. Obtenido de $:$ https://www.redalyc.org/articulo.oa? $\mathrm{id}=60426901002$

Mowday, R., Steers, R. M., y Porter, L. W. (1979). La medición del compromiso organizacional. Revista de comportamiento vocacional, pp. 224-247. Doi: 10.1016/0001-8791(79)90072-1

Navas, W. (2017). Investigación e innovación, factores de crecimiento en las PYMES. Revista Publicando, 4 (12), pp. 254-268. Obtenido de : https://revistapublicando.org/revista/index.php/crv/article/view/637

Nelson, R., \& Winter, S. (1982). The Schumpeterian trade-off revisited. American Economic Review.

Nijkamp, P., \& Ubbels, B. (1999). Infrastructure, suprastructure and ecostructure: a portfolio of sustainable growth potentials. Faculteit der Economische Wetenschappen en Econometric, Research Memorandum, pp. 1-18. Obtenido de: https://ideas.repec.org/p/vua/wpaper/1999-51.html

OCDE. (1996). La innovación tecnológica: definiciones y elementos de base . Redes, 3 (6), pp. 131-175. Obtenido de: https://www.redalyc.org/pdf/907/90711287005.pdf 
Olawale, \& Garwe. (2010). Obstacles to the growth of new SMEs in South Africa: A principal component analysis approach. African Journal of Business Management. African Journal of Business Management, 4(5), pp. 729738. https://academicjournals.org/article/article1380715803_Olawale\%20and\%20Garwe.pdf

OMC. (2016). Informe Anual de la Organización Mundial de Comercio. https://www.wto.org/spanish/res_s/booksp_s/anrep_s/anrep16_s.pdf: Organización Mundial de Comercio.

Ordóñez, J. (2015). Competitividad y bienestar en México: análisis de su relación con el. Madrid: Universidad Complutense de Madrid.

Peñaloza, M. (2007). Tecnológia e Innovación factores claves para la competitividad. Actualidad, 10 (15), pp. 82-94. Obtenido de: https://biblat.unam.mx/hevila/ActualidadcontableFACES/2007/vol10/no15/7.pdf

Perrotti, D., y Sánchez, R. (2011). La brecha de infraestructura en América Latina y el Caribe. Serie Recursos Naturales e Infraestructura, 153, pp. 85. Obtenido de: https://repositorio.cepal.org/handle/11362/6357

Piñeiro Chousa, J., y Romero Castro, N. (2001). El desarrollo sostenible en el sector energético: Las energías renovables en Galicia y su aportación al grupo unión Fenosa. Revista Galega de Economía, 10(2), pp. 1-23. Obtenido de: https://www.redalyc.org/pdf/391/39110214.pdf

Polimeni, R., Fabozzi, F., y Delberg, A. (1994). Contabilidad de Costos Conceptos y Aplicaciones para la Toma de Decisiones Gerenciales. McGraw-Hill.

Porter, M. (1990). The Competitive Advantage of Nations. The Free Press.

Porter, M. E. (2008). The Five Competitive Forces That Shape. Harvard business review, pp. 1-17. Obtenido de: https://hbr.org/2008/01/the-five-competitive-forces-that-shape-strategy

Prado, V. (2014). Evaluación de las Políticas Públicas del sector automotriz en el marco del TLCAN.

Prokopenko, J. (1989). La gestión de la productividad. Ginebra: OIT.

Quijano, S. (2006). Dirección de Recursos Humanos y Consultoría en Barcelona. Icaria.

Reyes, E., García, M., \& Herrera, J. (2014). Logística a la inversa. aprovechamiento de sobrantes, disminución de costos, reducción del impacto ambiental. Revista académica de economía. Obtenido de: https://www.eumed.net/cursecon/ecolat/mx/2014/logistica-inversa.html

Rickne, A., Laestadius, S., \& Etzkowitz, H. (2013). Innovation Governance in an Open Economy. Routledge.

Rivera, I., Ocampo, J., y Arredondo, L. (2011). El modelo de la triple hélice y la gestión de la vinculación en la Universidad Autónoma de Baja California. Universidad Autónoma de Baja California.

Rivera, R. F., \& Rivera, L. L. (2013). Ten strategies for strengthening university-industry linkage policies in Mexico. Journal of Teaching and Education, 5. https://www.academia.edu/36546510/TEN_STRATEGIES_FOR_STRENGTHENING_UNIVERSITY_INDU STRY_LINKAGE_POLICIES_IN_MEXICO?auto=download

Rodríguez, G., Balestrini, S., Balestrini, S., Meleán, R., \& Rodríguez, B. (2002). Análisis estratégico del proceso productivo en el sector industrial. Revista de Ciencias Sociales, 8 (1), pp. 135-156. Obtenido de: https://www.redalyc.org/pdf/280/28080109.pdf

Ros, J. (2004). El crecimiento económico en México y Centroamérica: desempeño reciente y perspectivas. Serie de estudios y perspectivas.

Rostow, W. (1967). Proceso de crecimiento económico. Alianza.

Saari, L., \& Judge, T. (2004). Employee attitudes and job satisfaction. Human Resource Management, 43 (4), pp. 395407. Doi: $10.1002 / \mathrm{hrm} .20032$

Saavedra, G. M. (2009). Problemática y desafíos actuales de la vinculación universidad empresa: El caso mexicano. 12 (19), pp. 21. Obtenido de: https://www.redalyc.org/pdf/257/25715409009.pdf

Samaniego, C. (1998). Absentismo, Rotación y Productividad. Introducción a la Psicología del trabajo y las organizaciones.Ediciones Pirámide.

Schiavon , J., \& Figueroa, B. (2019). Los recursos y capacidades de la Política Exterior de México (2012-2018). Foro Internacional, 59 (3), pp. 3-4. Doi: 10.24201/fi.v59i3-4.2635

Schumpeter, J. A. (1938). Business Cycles: A Theoretical, Historical and Statistical Analysis of the Capitalist Process. McGrawHill. 
Smallbone, D., \& Welter, F. (2001). The role of government in SME development in the transition economies of central and Eastern and the newly independent states. Croacia: Conference proceedings the fourth International Conference on Enterprise in Transition.

Sylos, L. (1993). Nuevas tecnologías y desempleo. México: Fondo de Cultura Económica.

Taaffe, E., Morril, R., \& Gould, P. (1963). Transport expansion in underdeveloped Countries: a comparative analysis. Geography Review, 53 (4), pp. 503-529. Doi: 10.2307/212383

Thompson, P., \& Strickland, G. (2012). Administración Estratégica. México: Mc Graw Hill.

Tolentino, A. (2004). New Concepts of Productivity and its Improvement. European Productivity Network Seminar, 37 (2) pp. 13-14. Obtenido de: https://www.redalyc.org/pdf/339/33922717007.pdf

Vargas, S. (18 de Febrero de 2020). Taller sobre la energía. Obtenido de Taller sobre la energía: https://issuu.com/svargassja/docs/taller_de_la_energia_santiago_vargas_s

Westreicher, G. (2 de Octubre de 2020). Economipedia. Obtenido de Economipedia: https://economipedia.com/definiciones/entorno-empresarial.html

Witker, J., \& Díaz, I. (Enero-Junio de 2011). El tratado de libre comercio de América del Norte. Defensa jurídica tardía del campo mexicano. Revista Latinoamericana de Derecho Social, (12), pp. 149-168. Obtenido de: https://www.redalyc.org/pdf/4296/429640267005.pdf

Ynzunza, C., \& Izar, J. (2013). Efecto de las estrategias competitivas y los recursos y capacidades orientados al mercado sobre el crecimiento de las organizaciones. Contaduría y Administración,58 (1), pp. 169-171. Obtenido de: https://www.redalyc.org/pdf/395/39525580008.pdf

Ynzunza, C., Márquez, A., \& Izar, J. (2008). El Efecto de las Fuerzas de Mercado en la Estrategia Competitiva, los Recursos y Capacidades Organizacionales y el Desempeño. Hitos de Ciencias Económico Administrativas, 58 (1), pp. 55-64. Doi: 10.1016/S0186-1042(13)71202-6

Zhao, M., Dröge, C., \& Stank, T. P. (2001). Los efectos de las capacidades logísticas en el desempeño de las empresas. Revista de logística empresarial, 22 (2), pp. 91-107. Doi: 10.1002/j.2158-1592.2001.tb00005.x 